Singh, B.

Kumar, S.

Sharma, M.K.

Patra, S.K.

exceptional evidence of atomic nucleus as a continuous source of novel as well as unexpected phenomenon.

The studies built on the "cold reaction valleys" have now led to the extension of the known Periodic Table of Elements up to $Z=118$ based on the concept of cold fusion. It was shown that the production of super heavy elements (SHE) need to choose suitable target-projectile combinations, and it is now accepted globally that the calculations of "cold reaction valleys" based on QMFT made possible the synthesis of all SHE.

The QMFT or the dynamical fragmentation theory is introduced briefly in Section II, followed by Section III with details on the phenomenon of CR and Section IV on the extension of Periodic Table to super heavy nuclei. The study is summarized in Section V. A brief report of this work was poster-presented at the DAE International Symposium on Nuclear Physics, BARC Mumbai, during December 2-6, 2013 [5].

\section{THE DYNAMICAL FRAGMENTATION THEORY}

The dynamical fragmentation theory or QMFT [1, 2], developed by Gupta and Collaborators, have been used to develop models of coupled motion in coordinates of relative separation $\mathrm{R}$ and mass asymmetry $\eta$, to study the ground state decay of nucleus and the decay of excited compound nucleus $(\mathrm{CN})$. The dynamics of such decays have been studied extensively in [6,7]. The Coulomb, nuclear proximity and the angular momentum dependent potentials are temperature dependent and include also the effects of deformations and orientations of the interacting nuclei. Thus, the ground state decay of nucleus as well as the decay of excited compound nucleus is worked out in terms of the following coordinates of the QMFT:

1. Relative separation coordinate R.

2. Deformation $\beta_{\lambda_{i}}$, orientations $\theta_{\mathrm{i}}(\mathrm{i}=1,2)$ of two nuclei.

3. A mass asymmetry coordinate $\eta=\left(A_{1}-A_{2}\right) /\left(A_{1}+A_{2}\right)$ and charge asymmetry coordinate $\eta_{\mathrm{Z}}=\left(\mathrm{Z}_{1}-\mathrm{Z}_{2}\right) /\left(\mathrm{Z}_{1}+\mathrm{Z}_{2}\right)$.

Then, in terms of these collective coordinates, for ground state decay ( $\mathrm{T}=0$ and $\mathrm{l}=0$ ) of nucleus, with in the preformed cluster model (PCM) [6], the decay constant $\lambda_{\mathrm{PCM}}$ is defined as (in terms of barrier assault frequency $\nu_{0}$, which is nearly constant $\sim 10^{21} \mathrm{~s}^{-1}$ )

$$
\lambda_{P C M}=\frac{\ln 2}{T_{1 / 2}}=v_{0} P_{0} P,
$$

and, using the partial wave analysis, for hot and rotating $(\mathrm{T} \neq 0$ and $\mathrm{l} \neq 0) \mathrm{CN}$, within the dynamical cluster-decay model (DCM) [7], the $\mathrm{CN}$ decay cross-section is defined as

$$
\sigma=\frac{\pi}{k^{2}} \sum_{l=0}^{l_{c}}(2 l+1) P_{0} P, \text { where } k=\sqrt{\frac{2 \mu E_{c . m}}{\hbar^{2}}}
$$


Here, the penetrability P, refers to the R-motion, given by the WKB integral

$$
P=\exp \left[-\frac{2}{\hbar} \int_{R_{a}}^{R_{h}}\left\{2 \mu\left[V(R)-Q_{e f f}\right]\right\}^{1 / 2} d R\right]
$$

with $\mathrm{V}\left(\mathrm{R}_{\mathrm{a}} \mathrm{T}\right)=\mathrm{V}\left(\mathrm{R}_{\mathrm{b}} \mathrm{T}\right)=\mathrm{TKE}(\mathrm{T})=\mathrm{Q}_{\text {eff }}$ corresponding to the first turning point or effective Q-value of the decay process, and $R_{a}=R_{1}(\eta, T)+R_{2}(\eta, T)+\Delta R(\eta, T)$, where $\Delta R(\eta, T)$ is the only parameter of the model $[6,7]$.

The pre-formation probability [6,7] is given by

$$
P_{0}=\sqrt{B_{\eta \eta}}\left|\psi\left[\eta\left(A_{i}\right)\right]\right|^{2}(2 / A)
$$

which is obtained by solving Schrödinger equation in $\eta$

$$
\left\{-\frac{\hbar^{2}}{2 \sqrt{B_{\eta \eta}}} \frac{\partial}{\partial \eta} \frac{1}{\sqrt{B_{\eta \eta}}} \frac{\partial}{\partial \eta}+V_{R}(\eta, T)\right\} \psi^{\nu}(\eta)=E^{\nu} \psi^{\nu}(\eta)
$$

where $\mathrm{V}_{\mathrm{R}}(\eta, \mathrm{T})$ is the fragmentation potential [7], defined as

$$
\begin{aligned}
V_{R}(\eta, T)= & \sum_{i=1}^{2}\left[V_{L D M}\left(A_{i}, Z_{i}, T\right)\right]+\sum_{i=1}^{2}\left[\delta U_{i}\right] \exp \left(-T^{2} / T_{0}^{2}\right) \\
& +V_{c}\left(R, Z_{i}, \beta_{\lambda i}, \theta_{i}, T\right)+V_{P}\left(R, A_{i}, \beta_{\lambda i}, \theta_{i}, T\right)+V_{l}\left(R, A_{i}, \beta_{\lambda i}, \theta_{i}, T\right)
\end{aligned}
$$

$\mathrm{V}_{\mathrm{c}}, \mathrm{V}_{\mathrm{P}}$ and $\mathrm{V}_{l}$ are, respectively, the temperature-dependent Coulomb, nuclear proximity and angular momentum dependent potentials.

\section{THE FOURTH KIND OF NATURAL RADIOACTIVITY}

\section{A. Cluster Radioactivity and PCM}

Cluster Radioactivity (CR) is the spontaneous emission of clusters, heavier than $\alpha$-particle; also, sometimes referred to as "magic radioactivity" because cluster decays in trans-Lead region have always a doubly magic daughter nucleus ${ }^{208} \mathrm{~Pb}$ ( $Z=82, N=126$ ) or a nucleus close to it. One may also relate this phenomenon to $\alpha$-decay, in which a much lighter doubly magic nucleus ${ }^{4} \mathrm{He}(\mathrm{Z}=2, \mathrm{~N}=2)$ is emitted, and thus term it as Pb-radioactivity. The theoretical prediction of $\mathrm{CR}$ was actually based on fragmentation potentials or cold valleys (calculated by using the QMFT by Gupta and Collaborators) of those binary decays containing one partner as ${ }^{208} \mathrm{~Pb}$ or close to it (see Fig. 1 of Ref. [8]). The cluster-decay half-lives (and its branching ratios with respect to $\alpha$ decay) for spontaneous emission of some 13 to 14 heavy clusters, ranging from ${ }^{14} \mathrm{C}$ up to ${ }^{34} \mathrm{Si}$, are measured for the ground-state decays of certain parent nuclei in trans-Lead region, specifically from ${ }^{221} \mathrm{Fr}$ to ${ }^{242} \mathrm{Cm}[9,10]$, including also the recent observations of ${ }^{14} \mathrm{C}$ and ${ }^{15} \mathrm{~N}$ decays of ${ }^{223} \mathrm{Ac}$ and ${ }^{34} \mathrm{Si}$ decay of ${ }^{238} \mathrm{U}$ [11].
Extensions of Natural Radioactivity to 4th-Type and of the Periodic Table to Super-heavy Nuclei: Contribution of Raj

K Gupta to Cold Nuclear Phenomena 
Singh, B.

Kumar, S.

Sharma, M.K.

Patra, S.K.

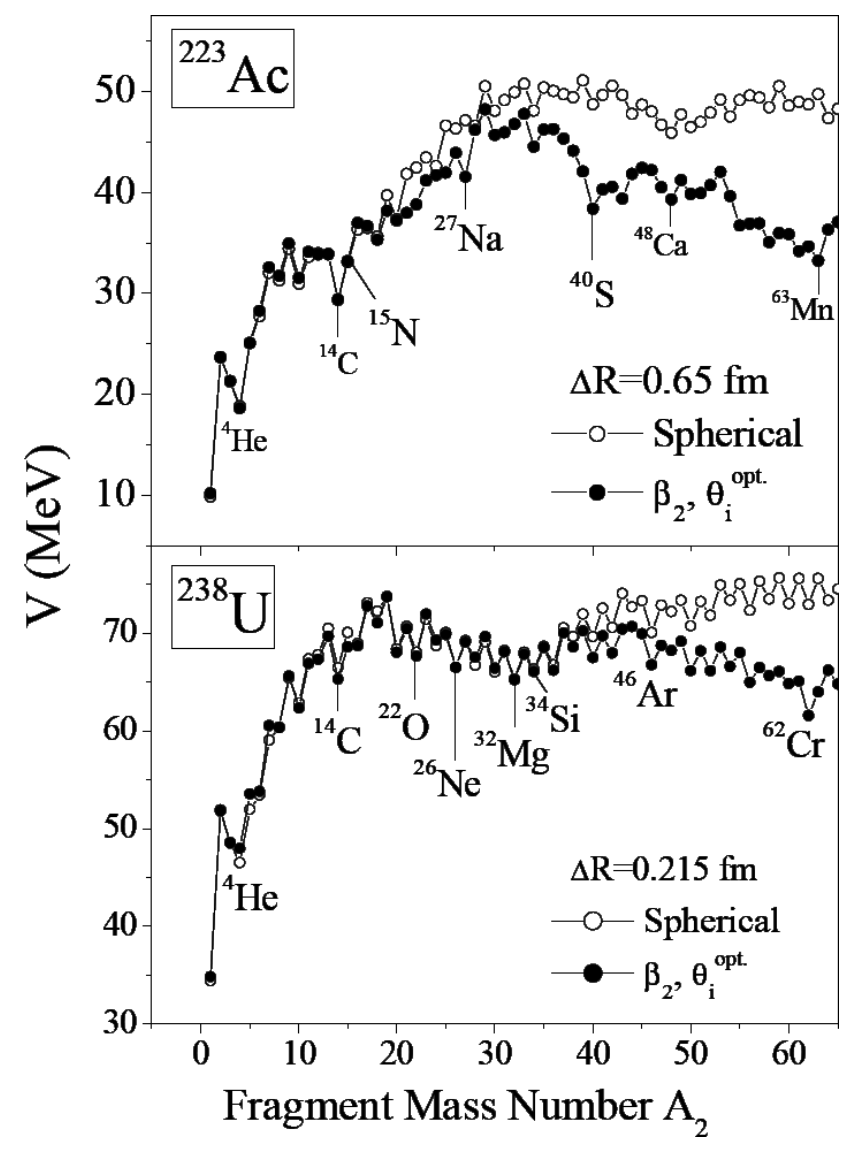

Figure 1: The fragmentation potentials for the parent nuclei ${ }^{223} \mathrm{Ac}$ (upper panel) and ${ }^{238} \mathrm{U}$ (lower panel), taking all possible fragments as spheres (open circles) and with quadrupole deformation $\beta_{2}$ and cold "optimum"

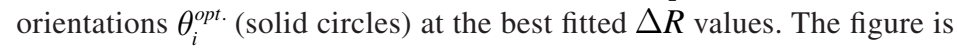
based on Fig. 2 of PRC 80, 034317(2009).

To understand the CR phenomenon, the unified fission models (UFM) such as the analytic super-asymmetric fission model (ASAFM) [3], and PCM like that of Gupta and Collaborators [6] have been advanced. They differ from each other for their noninclusion or inclusion of the preformation probability/ spectroscopic factor $\mathrm{P}_{0}$ of the cluster being preformed before penetrating the confining nuclear interaction barrier with certain penetration probability $\mathrm{P}$.

The ${ }^{14} \mathrm{C}$ and ${ }^{15} \mathrm{~N}$ decays of ${ }^{223} \mathrm{Ac}$ and ${ }^{34} \mathrm{Si}$ decay of ${ }^{238} \mathrm{U}$ have also been studied by Gupta and collaborators [6] recently, within PCM. Fig. 1 gives thefragmentation potentials for the parent nuclei ${ }^{223} \mathrm{Ac}$ and ${ }^{238} \mathrm{U}$. Here, all possible fragments are 


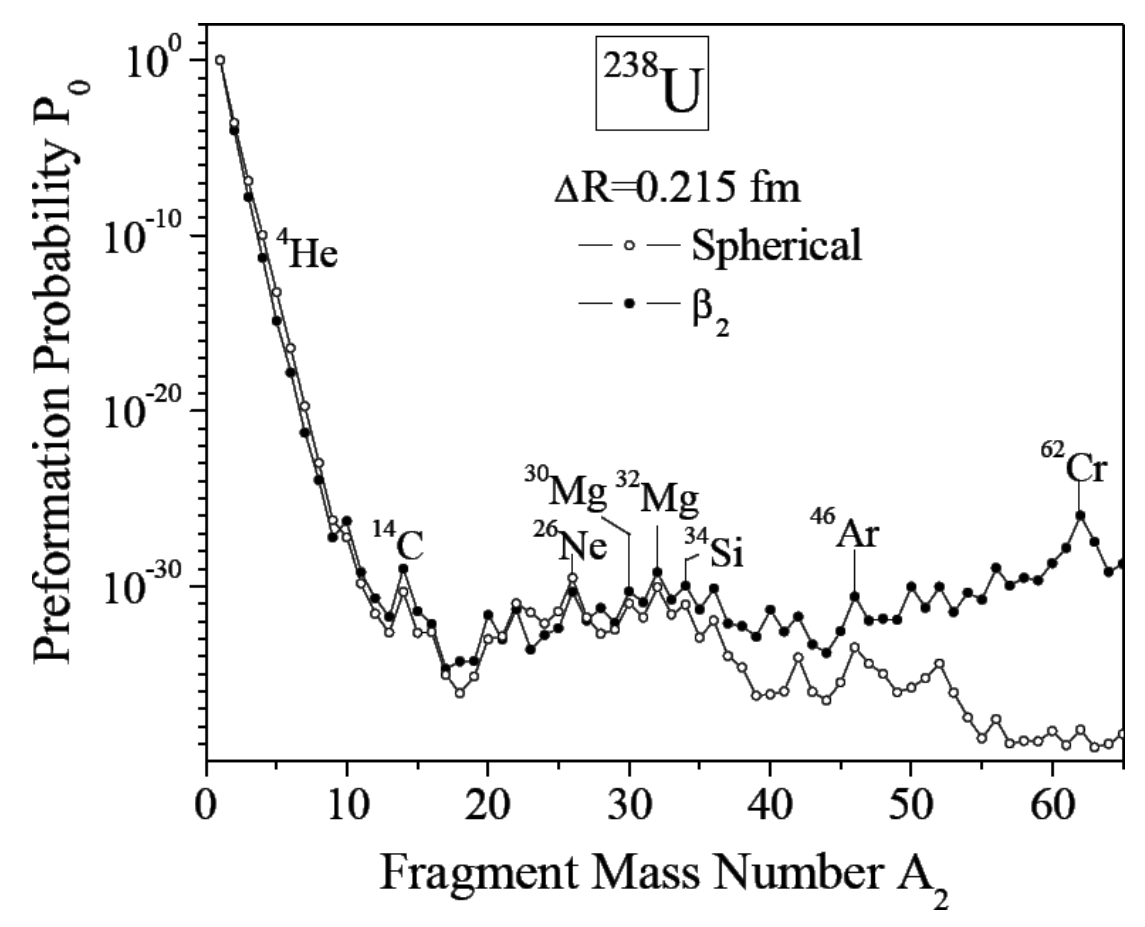

Figure 2: Same as for Fig. 1(lower panel, ${ }^{238} \mathrm{U}$ ), but for the preformation probability $\mathrm{P}_{0}$. The figure is based on Fig. 3 of PRC 80, 034317 (2009).

considered as spheres (open circles) and, then, with quadrupole deformation $\beta_{2}$

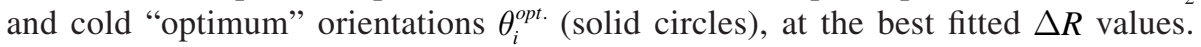
In Fig. 2 the preformation probability, $\mathrm{P}_{0}$, for ${ }^{238} \mathrm{U}$ have been calculated. In these calculations [6], experimental data [11] have been compared nicely, while discussing the dynamics of the decay using PCM.

\section{B. Cluster Radioactivity and RMFT}

In PCM, for the first time, Gupta and collaborators assumed the clusters to be preborn in the parent nucleus with certain $\mathrm{P}_{0}$ 's, calculated by solving the stationary Schrödinger equation for the dynamical flow of mass and charge, the only available method to-date to calculate $\mathrm{P}_{0}$. It may be noted here that the mass and charge densities calculated by using the Relativistic Mean Field Theory (RMFT) are also known to support the clustering effects in various heavy parents with observed cluster decays [12]. The total matter density distributions for the ground and intrinsic excited states of radioactive nuclei ${ }^{222} \mathrm{Ra},{ }^{232} \mathrm{U},{ }^{236} \mathrm{Pu}$ and ${ }^{242} \mathrm{Cm}$, for use of the NL3 parameter set in the RMF calculation are presented in Fig. 3 of Ref. [12]. The dark-blue contours refer to maximum density and the dark-red ones to
Extensions of

Natural Radioactivity to 4th-Type and of the Periodic Table to Super-heavy Nuclei: Contribution of Raj K Gupta to Cold Nuclear Phenomena 
Singh, B.

Kumar, S.

Sharma, M.K.

Patra, S.K.

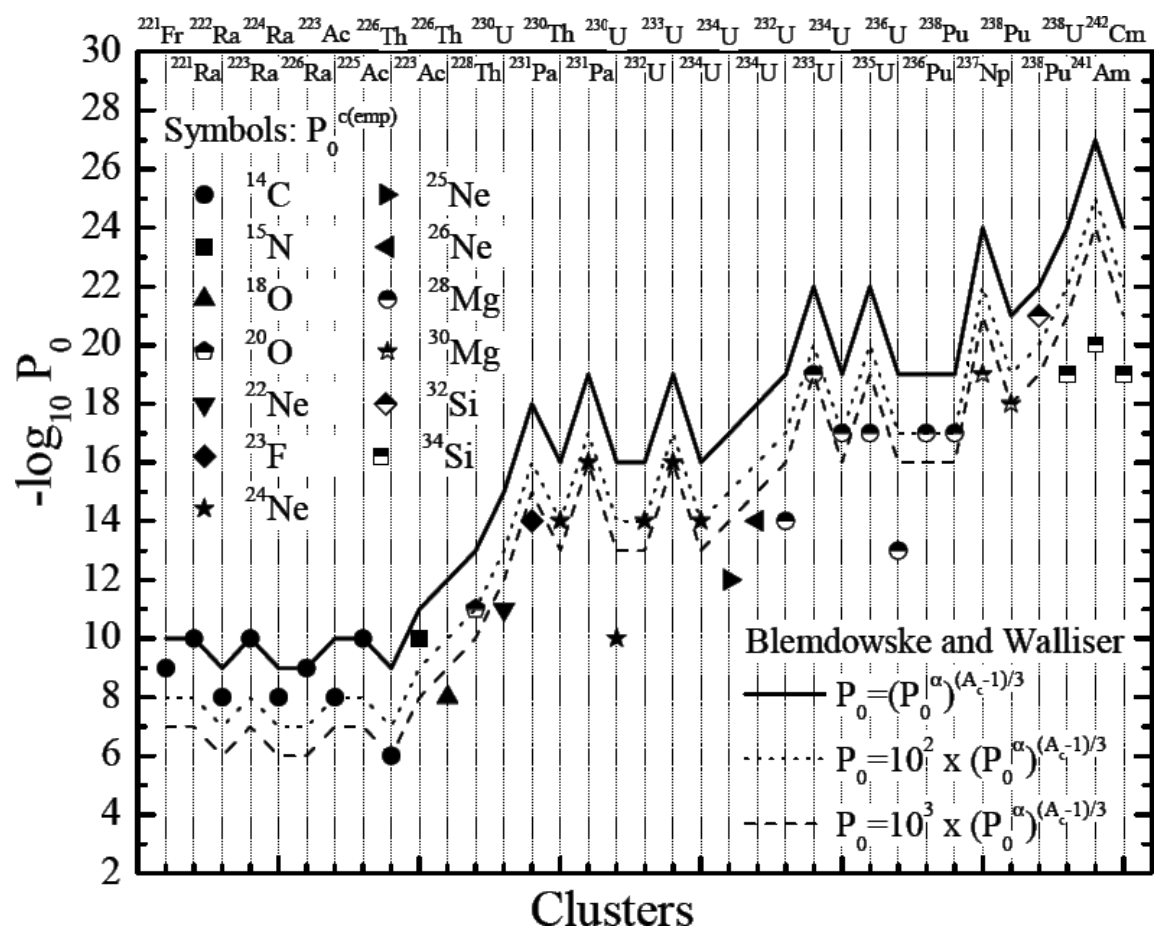

Figure 3: The empirical preformation probability $P_{0}^{c(e m p)}$ for cluster decays from various parents (symbols) compared with the model calculations of Blendowske and Walliser[13] (solid line) and ones raised by the factors of $10^{2}$ and $10^{3}$ (dotted and dashed lines, respectively). The figure is based on Fig. 2 of PRC 82, 014607(2010).

zero density. We note in this figure that the low-density regions (referred to as clusters) occur in each case, for both the ground- and excited-state solutions. The important point is that the microscopic relativistic mean field (RMF) formalism, combined with an effective M3Y nucleon-nucleon interaction, supports the concept of preformation of clusters in nuclei, introduced by Gupta and collaborators in PCM for cluster radioactive decays. In Fig. 3 , the $\mathrm{P}_{0}$ (emp) values from various parents (symbols) compared with the model calculations of Blendowske and Walliser [13] (solid line), and ones raised by the factors of $10^{2}$ and $10^{3}$ (dotted and dashed lines), respectively, quite reasonably.

\section{EXTENSION OF THE PERIODIC TABLE TO SUPER HEAVY NUCLEI}

The study of "cold reaction valleys" by Prof. Raj K. Gupta and collaborators, almost forty years ago, led to an idea of cold fusion (reaction partners) and succeeded in 


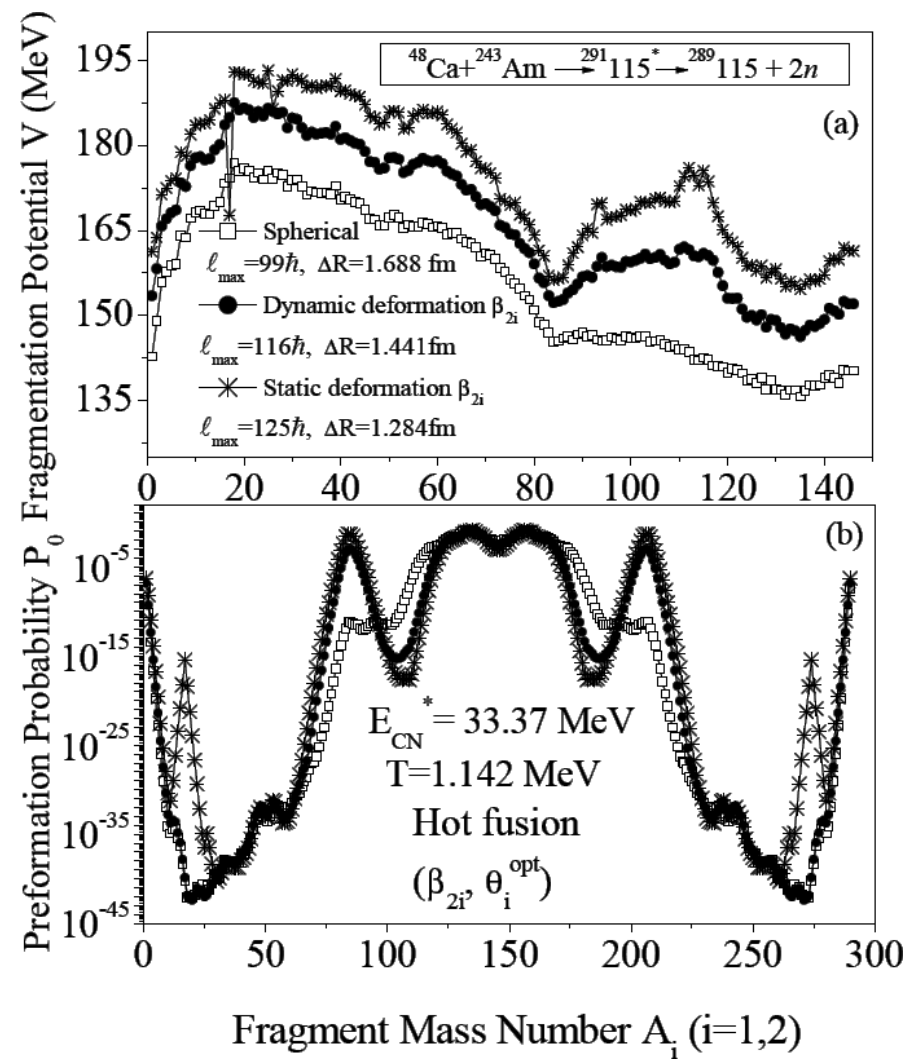

Extensions of

Natural Radioactivity

to 4th-Type and of

the Periodic Table to

Super-heavy Nuclei:

Contribution of Raj

K Gupta to Cold

Nuclear Phenomena

Figure 4(a): Fragmentation potential for the decay of ${ }^{291} 115^{*}$ compound system formed in the ${ }^{48} \mathrm{Ca}+{ }^{243} \mathrm{Am}$ reaction for $\theta_{i}^{\text {opt. }}$ at $E_{C N}^{*}=33.37 \mathrm{MeV}$ and respective $l_{\max }$ values. (b) Same as for (a), but for the corresponding preformation probabilities $\mathrm{P}_{0}$. The figure is based on Fig.2 of PRC 87, 054610 (2013).

extending the known periodic table of elements up to $\mathrm{Z}=118$. It was predicted that for production of nuclei with $\mathrm{Z} \geq 102$ there is a necessity to bombard suitable projectile on doubly magic nuclei and it was further shown that the most favorable combinations are related to the valley of heavy cluster emission, i.e., the so called $\mathrm{Pb}$ potential valley. It is recognized world-wide that these predictions made possible the production of all SHE $Z \leq 118$ in Darmstadt and Dubna [14-16], experimentally (since 1994), based on the idea of cold reaction valleys. Furthermore, Gupta and collaborators have developed and used DCM [7] to study the decay of excited compound nuclei as a collective clusterization process for emission of the light particles LPs, intermediate mass fragments IMFs and heavy mass fusion-fission fragments FFs, in contrast to statistical models in which each type of emission is treated on different footing. In Fig. 4(a) we have shown the fragmentation potential for the decay of ${ }^{291} 115^{*}$ compound 
Singh, B.

Kumar, S.

Sharma, M.K.

Patra, S.K.

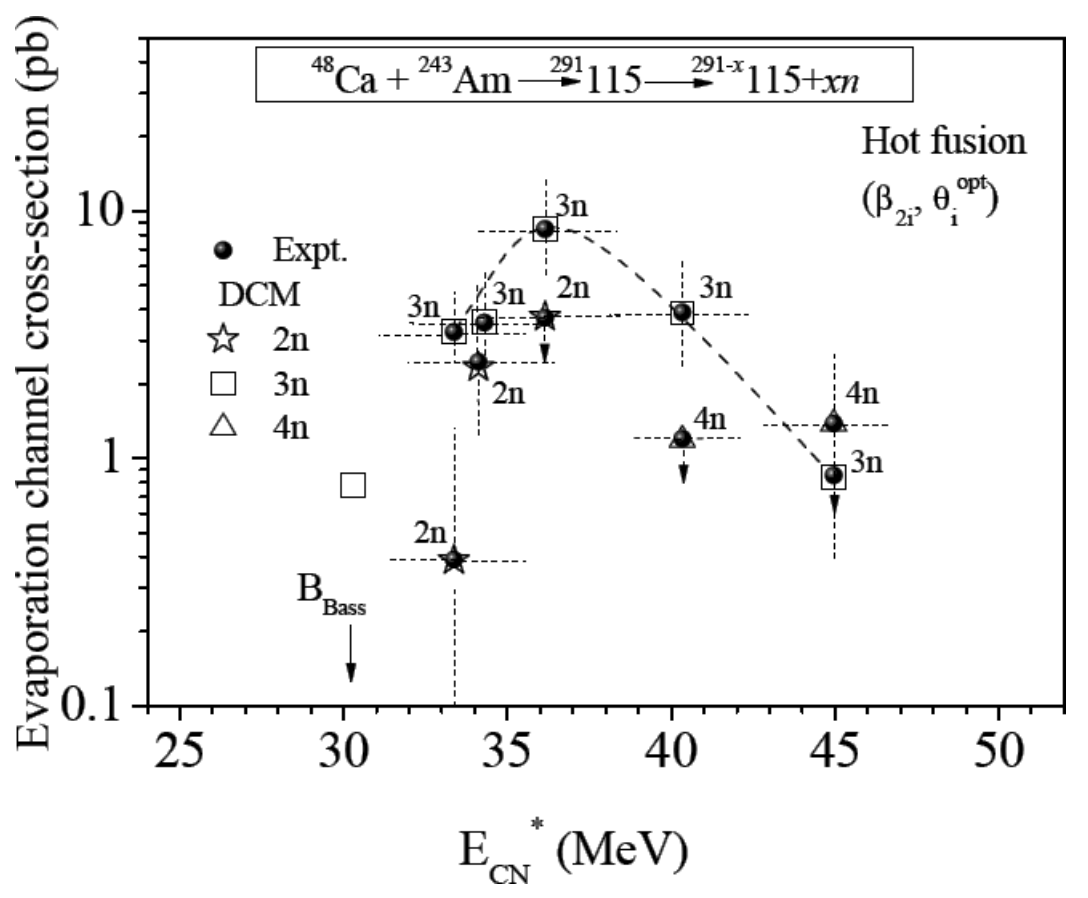

Figure 5: Calculated cross sections for $2 n$-, $3 n$-, and $4 n$-evaporation channels of ${ }^{48} \mathrm{Ca}+{ }^{243} \mathrm{Am}$ reaction as a function of $E_{C N}^{*}$, compared with experimental data [16]. The error bars are also shown. The figure is based on Fig. 6 of PRC 87, 054610 (2013).

system formed in the ${ }^{48} \mathrm{Ca}+{ }^{243} \mathrm{Am}$ reaction at $E_{C N}^{*}=33.37 \mathrm{MeV}$ calculated by Gupta and Collaborators. Here calculations have been made for spherical and deformed choices of fragments with optimum hot orientations $\theta_{i}^{\text {opt. }}$ and respective $l_{\max }$ values of $99 \hbar$ and $116 \hbar$, respectively. The deformations are taken up to quadrupole $\beta_{2 i}$. In Fig. 4(b) corresponding preformation probabilities $\mathrm{P}_{0}$ have been calculated. The calculations for the cross sections for $2 n-, 3 n-$, and $4 n$-evaporation channels of the reaction as a function of $E_{C N}^{*}$ have been compared with experimental data [16], as shown in Fig. 5. The error bars are also shown here along with dashed line as guide to the eye. Similar calculations are shown in Fig. 6 and Fig. 7 but for ${ }^{48} \mathrm{Ca}+{ }^{249} \mathrm{Bk}$ $\rightarrow{ }^{297} 117^{*}$ reaction.

\section{SUMMARY}

The idea of "cold reaction valleys" based on QMFT, led to the prediction and verification of very rare (fourth) type of natural radioactivity along with the extension of periodic table. It may be emphasized that QMFT alone gives unified description 


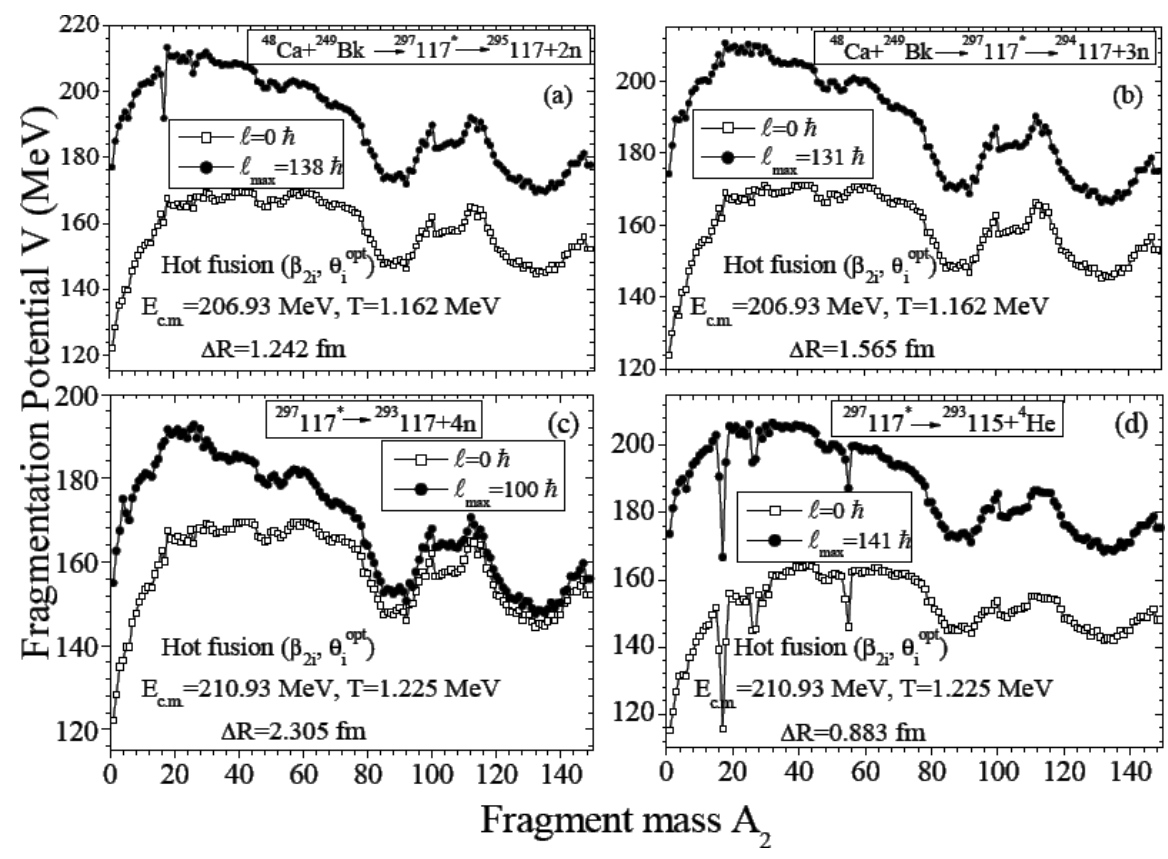

Extensions of Natural Radioactivity to 4th-Type and of the Periodic Table to Super-heavy Nuclei: Contribution of Raj K Gupta to Cold Nuclear Phenomena

Figure 6(a-d) Fragmentation potentials for ${ }^{48} \mathrm{Ca}+{ }^{249} \mathrm{Bk} \rightarrow{ }^{297} 117 *$ for various neutron-evaporation channel including the ${ }^{4} \mathrm{He}$ decay channel. The figure is based on Fig.3 of PRC 85, 024604 (2012).

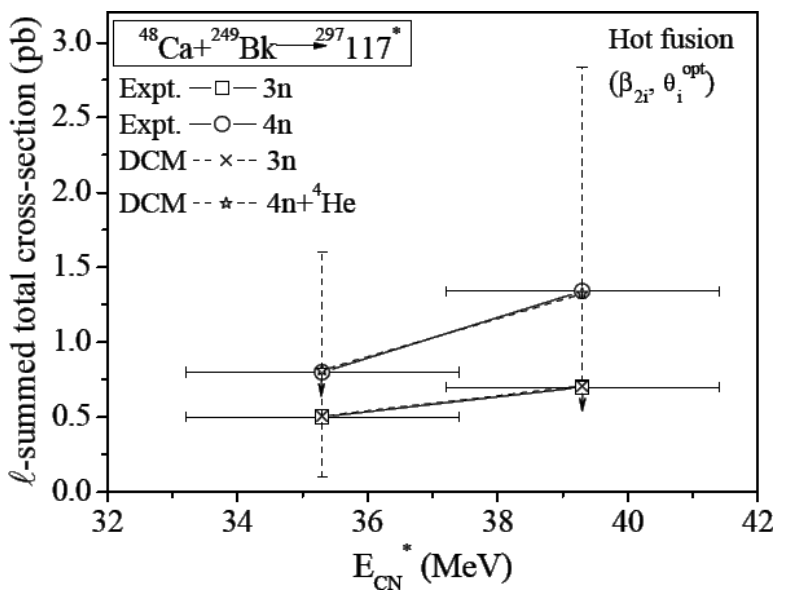

Figure 7: DCM calculated cross sections $\sigma_{3 \mathrm{n}}$ and $\sigma_{4 \mathrm{n}+4 \mathrm{He}}$ for the decay of ${ }^{297} 117^{*}$, plotted as a function of $E_{C N}^{*}$ and compared with experimental data [15]. The figure is based on Fig. 8 of PRC 85, 024604 (2012). 
Singh, B.

Kumar, S.

Sharma, M.K.

Patra, S.K. for all the three cold processes: cluster radioactivity, cold fission and cold fusion. It also led to the development of dynamical theories (PCM and DCM) to successfully explain the ground-state as well as excited-state decay of nuclei. The DCM has been applied successfully to the decay of light, medium, heavy and super heavy mass compound nuclei. Here, a concept of preformed clusters in nuclei lead to a nonstatistical description for the decay of a CN to light particles LPs (n, p and $\alpha$-particle), the intermediate mass fragments IMFs (both light and heavy), fusion-fission FF and quasi-fission QF processes. Different mass regions of the Periodic Table show different combinations of these processes or any one of them as a dominant mode and DCM seems to provide a decent description of these decay modes. It is highly relevant to mention here that cold fission was also predicted on QMFT in 1975, but first observed in 1981. The whole concept of cold phenomenon in nuclear physics was first introduced by Prof. Raj K. Gupta and collaborators on the basis of QMFT. These are among the most substantial accomplishments of Indian Physics, and we owe them to Prof. Raj K. Gupta who celebrates his $75^{\text {th }}$ birthday this year, dynamic as ever.

\section{REFERENCES}

[1] H. J. Fink, et al., Proc. Int. Conf. on Reactions between Complex Nuclei, Nashville, USA, June 10-14, 2, 21, (1974); Raj K. Gupta, et al., Proc. Int. School on Nucl. Phys., Predeal, Romania, 75, 1974; J. Maruhn and W. Griener, Phys. Rev. Lett. 32, 548, (1974). http://dx.doi.org/10.1103/PhysRevLett.32.548

[2] Raj K. Gupta, et al., Phys. Rev. Lett. 35, 353, (1975); Phys. Lett. 60 B, 225, (1976); Phys. Lett. 67B, 257, (1977); Z. Naturforsch 32a, 704, (1977); Z. Physik A 283, 217, (1977); Sovt. Jour. Nucl. Phys. 28, 160, (1978); Z. Physik A 281, 159, (1977); Sovt. Jour. Nucl. Phys. 8, 289, (1977); Fiz. Elem. Chastis. At. Yadra 8, 717, (1977). http://dx.doi.org/10.1103/PhysRevLett.35.353

[3] A. Săndulescu, et al., Sovt. Jour. Nucl. Phys. 11, 528, (1980).

[4] H. J. Rose and G. A. Jones, Nature 307, 245, (1984). http://dx.doi.org/10.1038/307245a0

[5] BirBikram Singh, Manoj K. Sharma and S. K. Patra, in Proceedings of the Int. DAE Symp. on Nucl. Phys. 58, 450, (2013).

[6] Raj K. Gupta, et al., in Proc. of the 5th Int. Conf. on Nuclear Reaction Mechanisms, Varenna, (Ricerca Scientificaed Educazione Permanente, Milano, 1988), p. 416; Phys. Rev. C 39, 1992, (1989); Phys. Rev. C 55, 218, (1997); Phys. Rev. C 79, 064616, (2009); Phys. Rev. C 80, 034317, (2009); Phys. Rev. C 82, 014607, (2010); Int. J. Mod. Phys. E 20, 1003, (2011). http://dx.doi.org/10.1142/S0218301311019143

[7] Raj K. Gupta et al., Int. Rev. Phys (IREPHY) 2, 369, (2008); Clusters in Nuclei, Lecture Notes in Physics, 818,223, (2010), ed. C. Beck, Springer-Verlag; Proc. DAE Symp. OnNucl. Phys. 56, 474, (2011); 57, 550, (2012); Phys. Rev. C 85, 064602, (2012), 85, 024615, (2012); Phys. Rev. C 85, 024604, (2012); Phys. Rev. C 86, 034604, (2012); Phys. Rev. C86, 064610, (2012); Phys. Rev. C 86, 064611, (2012); Phys. Rev. C 87, 054610, (2013); Phys. Rev. C 88, 014615, (2013). http://dx.doi.org/10.1103/PhysRevC.88.014615

[8] Raj K. Gupta, in Heavy Elements and Related New Phenomena, World Scientific, Singapore, Vol. II, p. 643, (1999). 
[9] Raj K. Gupta and W. Greiner, Int. J. Mod. Phys. E3, 335, (1994). http://dx.doi.org/10.1142/S0218301394000127

[10] R. Bonetti and A. Guglielmetti, Romanian Reports in Physics 59, 301, (2007).

[11] A. Guglielmetti, et al., J. Phys.: Conf. Ser. 111, 012050, (2008). http://dx.doi.org/10.1088/1742-6596/111/1/012050

[12] S. K. Patra, et al., J. Phys. G: Nucl. Part. Phys. 34, 2073, (2007). http://dx.doi.org/10.1088/0954-3899/34/9/016

[13] R. Blendowske and H. Walliser, Phys. Rev. Lett. 61, 1930, (1988). http://dx.doi.org/10.1103/PhysRevLett.61.1930

[14] S. Hofmann, et al., Nucl. Phys. News 14, No. 4, 5, (2004). http://dx.doi.org/10.1080/10506890491034938

[15] Yu. Ts. Oganessian et al., Nucl. Phys. A 734, 109, (2004); Phys. Rev. C 83, 054315, (2011). http://dx.doi.org/10.1016/j.nuclphysa.2004.01.020

[16] Yu. Oganessian et al., Phys. Rev. Lett. 108, 022502, (2012); Phys. Rev. C 87, 014302, (2013). http://dx.doi.org/10.1103/PhysRevLett.108.022502 